\title{
Evaluation of Smear Layer after Er:YAG Laser Irradiation in Middle and Apical Third of Mesial Root Canals: A Comparative SEM Investigation
}

\author{
Evrykleia Kourti ${ }^{1}$, Ourania Pantelidou-Papadopoulou ${ }^{2}$, Kosmas Tolidis $^{3}$, Dimitris Strakas ${ }^{4}$
}

\begin{abstract}
Aim and objective: This ex vivo study evaluated the efficiency of an Er:YAG laser ( $2940 \mathrm{~nm}$ ) at different pulse energy levels to remove the smear layer with or without chelators from the middle and apical third of mesial roots.

Materials and methods: Thirty-four mesial root canals of first mandibular molars (type II Vertucci) were divided into four groups of eight teeth each. Each group consisted of two subgroups each ( $A$ and $B$ ), regarding the irrigation protocol. In subgroup $A$, teeth were rinsed only by distilled water whereas, in subgroup B, teeth were rinsed by $5 \mathrm{~mL} 17 \%$ EDTA for 60 seconds, $5 \mathrm{~mL} 5 \% \mathrm{NaOCL}$, and $5 \mathrm{~mL}$ distilled water. The rest two teeth were used as control groups. After coronal access, all teeth were instrumented up to size F3 (30/0.09) and, then, the experimental groups were irradiated by an Er:YAG laser (2940 nm). Four different pulse energy values were tested, namely 30, 50, 70, and $80 \mathrm{~mJ}$ to irradiate the roots in group I, group II, group III, and group IV, respectively. The control group $(n=2)$ was instrumented and rinsed as experimental groups (subgroup B) but not irradiated. Teeth were observed under SEM. Results were statistically analyzed with the Kruskal-Wallis test.

Results: There is a statistically significant difference between groups irrigated with chelators and groups with distilled water before laser irradiation in the apical third. Group IB $(0.75 \mathrm{~W})$ showed a statistically significant outcome in the apical part. The results showed no statistical difference between subgroup $B$ and the control group.

Conclusion: The presence of a chelating factor may play an important role in the laser mechanism of smear layer removal from the apical part of narrow and curved root canals.

Keywords: Er:YAG laser, Mesial root canals, Molars, Smear layer removal.

International Journal of Experimental Dental Science (2021): 10.5005/jp-journals-10029-1221
\end{abstract}

\section{INTRODUCTION}

The absorption of laser light is strongly dependent on the laser wavelength and kind of tissue. Mid-infrared wavelengths between 2.7 and $3 \mu \mathrm{m}$ exhibit a high absorption in water and hydroxyapatite, thus making them highly effective in ablating hard dental tissues. ${ }^{1,2}$ Currently, the most common lasers of the mid-infrared spectrum used in dentistry, are the Er:YAG laser ( $2940 \mathrm{~nm}$ ) and the Er,Cr:YSGG laser $(2790 \mathrm{~nm}) .^{3}$

Specifically, Er:YAG laser has the highest absorption in water with an absorption coefficient $\mu \mathrm{A}$ of $800 \mathrm{~cm}^{-1}$. Considering that hard dental tissues consist of water in different percentages, ${ }^{4} \mathrm{Er}: Y A G$ laser can ablate them. ${ }^{5,6}$ Therefore, Er:YAG laser is an effective tool for smear layer removal in root canals. ${ }^{7-10}$

Dental hard tissue removal can be achieved by two mechanisms either thermo-mechanical ablation or explosive vaporization. ${ }^{11,12}$ In Erbium lasers, the smear layer is removed through the process of thermo-mechanical ablation. ${ }^{12}$

Understanding the morphology of the root canal is of primary importance in achieving the long-term success of the treatment. ${ }^{13}$ Anatomically permanent mandibular first molar typically displays a mesial and a distal root with two mesial and one distal canal. ${ }^{14-16}$ The most common canal configuration in mesial roots of first is type IV and type II (31.5-28\%). ${ }^{17}$

The study had a purpose to evaluate smear layer removal in the middle and apical third of mesial root canals irradiated by an Er:YAG laser and determining the optimal values of pulse energy and average power. Therefore, Er:YAG laser, using a conical designed
'Department of Dental School, Aristotle University of Thessaloniki, Aghios Stefanos, Greece

${ }^{2}$ Department of Endodontics, Dental School of Aristotle University of Thessaloniki, Thessaloniki, Greece

${ }^{3}$ Department of Operative Dentistry, Aristotle University of Thessaloniki, Thessaloniki, Greece

${ }^{4}$ Department of Operative Dentistry, University of Thessaloniki, Thessaloniki, Greece

Corresponding Author: Evrykleia Kourti, Department of Dental School, Aristotle University of Thessaloniki, Aghios Stefanos, Greece, Phone: +30-6936779437, e-mail: evakourti@hotmail.com

How to cite this article: Kourti E, Pantelidou-Papadopoulou O, Tolidis $\mathrm{K}$, et al. Evaluation of Smear Layer after Er:YAG Laser Irradiation in Middle and Apical Third of Mesial Root Canals: A Comparative SEM Investigation. Int J Experiment Dent Sci 2021;10(1):14-18.

Source of support: Nil

Conflict of interest: None

fiber tip can effectively remove the smear layer in the apical root third, without the aid of any chemical irrigants.

\section{Materials and Methods}

Research Ethics Committee of Dental School of the Aristotle University of Thessaloniki approved the protocol (no 363/16.01.18). A total number of 34 freshly extracted mandibular first molars with 
two roots were used. The mesial root was separated from the distal and was placed in a holder. Cone-beam computed tomography (CBCT) was used for scanning and separation of roots according to the configuration anatomy. Mesial roots, joining to common apical foramen (type II Vertucci classification), were collected.

Root canals were instrumented using Protaper Gold (DentsplyMaillefer, Ballaigues, Switzerland) up to size F3 (30/0.09) and irrigated with $1 \mathrm{~mL}$ distilled water between instrumentations. At the end of the process, teeth were randomly assigned into four groups with two subgroups each namely $A$ and $B$, depending on the protocol used to remove the smear layer. In subgroup $A$, teeth were rinsed only by $5 \%$ distilled water whereas, in subgroup $B$, teeth were rinsed by $5 \mathrm{~mL} 17 \%$ EDTA for 60 seconds, $5 \mathrm{~mL} 5 \% \mathrm{NaOCL}$, and $5 \mathrm{~mL}$ distilled water. Teeth were incubated at $37^{\circ} \mathrm{C}$ temperature and $100 \%$ humidity until use.

An Er:YAG laser system (2940 nm) (Morita AdvErl Evo, Kyoto, Japan) was used. The tip utilized was a $300 \mu \mathrm{m}$ diameter radial tip (R300T), applied in a suitable handpiece (Morita, N8001736) (Fig. 1). The laser tip was inserted into the root canal $1 \mathrm{~mm}$ short of the apex. The movement was longitudinal circular movement from the apical to the coronal part of the root. The speed of the movement was $2 \mathrm{~mm} / \mathrm{s}$. Each root canal was irradiated four times.

The samples were irradiated according to the following protocol (Table 1):

- In group I, the laser parameters set were: pulse energy $30 \mathrm{~mJ}$, the average output power of $0.75 \mathrm{~W}$, the pulse repetition rate of $25 \mathrm{~Hz}$, pulse duration of $300 \mu \mathrm{s}$, and the 7:7 water/air ratio.

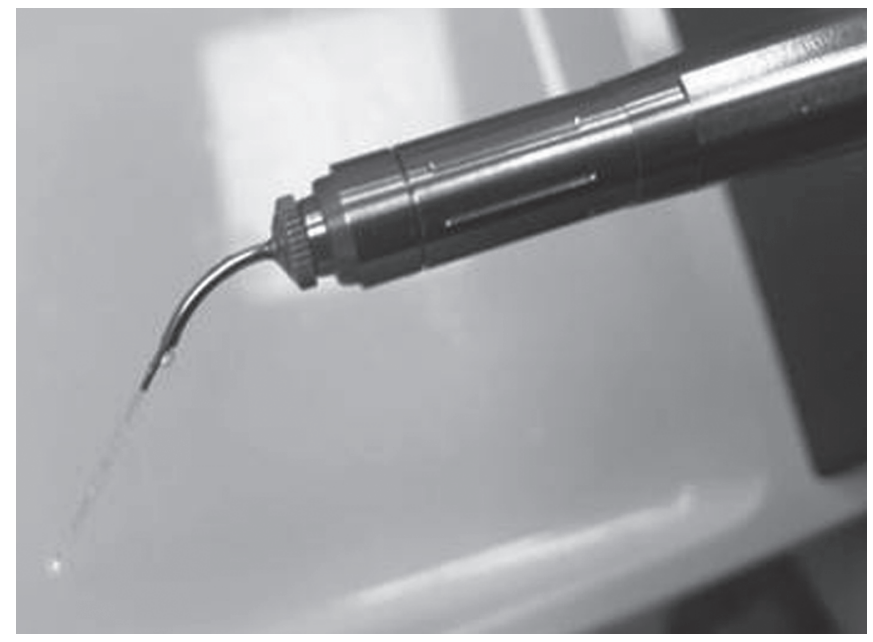

Fig. 1: Tip Er:YAG laser R300T (Morita Corporation)
- In group II, the laser parameters set were: pulse energy $50 \mathrm{~mJ}$, the average output power of $1.25 \mathrm{~W}$, the pulse repetition rate of $25 \mathrm{~Hz}$, pulse duration of $300 \mu \mathrm{s}$, and the $7: 7$ water/air ratio.

- In group III, the laser parameters set were: pulse energy $70 \mathrm{~mJ}$, the average output power of $1.75 \mathrm{~W}$, the pulse repetition rate of $25 \mathrm{~Hz}$, pulse duration of $300 \mu \mathrm{s}$, and the 7:7 water/air ratio.

- In group IV, the laser parameters set were: pulse energy $80 \mathrm{~mJ}$, the average output power of $2 \mathrm{~W}$, the pulse repetition rate of 25 $\mathrm{Hz}$, pulse duration of $300 \mu \mathrm{s}$, and the $7: 7$ water/air ratio.

- In the control group $(n=2)$, teeth were instrumented and rinsed as the experimental groups (subgroup B). No laser irradiation was performed.

All sample irradiations were performed by a single operator. After irradiation, two longitudinal grooves were made on the outer root surface. Teeth were split along their long axis in a buccolingual direction. From the two halves, only the mesial half of the mesial root was selected and the distal part was discarded. Mesial parts were observed under the scanning electron microscope at 2,000x magnification. Digital images were taken from the common apical third of the buccal and lingual root canal.

The percentage of smear layer residues was estimated according to the rating system below:

- $0: 0 \%$ residues.

- $1:<20 \%$ residues.

- 2: $20-50 \%$ residues.

- $3:<50 \%$ residues.

Two calibrated examiners evaluated the number of residues. A third examiner assisted in the scoring of the sample in case of disagreement.

The Cohen's $\kappa$ coefficient was used to analyze the inter-rater agreement. Results were statistically analyzed with Kruskal-Wallis and Mann-Whitney test. Statistical analysis was performed with SPSS Statistics Software and the significance level was set at $5 \%$ $(p<0.05)$.

\section{Results}

The Cohen $\kappa$ analysis showed excellent reliability and reproducibility between the evaluators with values $\geq 1$ for both. Different results were observed in experimental groups while comparing different laser parameters and irrigation protocols applied. The results in the middle and apical third of the mesial root canals in each group are shown in Tables 2 and 3, respectively.

More specifically, samples of groups IA, IIA, IIIA, and IVA, were irrigated only by distilled water. In the apical part of the root canal, SEM pictures showed the presence of a heavy and intact smear layer.

Table 1: Experimental groups

\begin{tabular}{llllllll}
\hline & Groups & Average power & Pulse energy & Repetition rate & Pulse duration & Water/air ratio & Irrigation \\
\hline Group I & IA $(n=4)$ & $0.75 \mathrm{~W}$ & $30 \mathrm{~mJ}$ & $25 \mathrm{~Hz}$ & $300 \mu \mathrm{s}$ & $7: 7$ & $\mathrm{dw}$ \\
& IB $(n=4)$ & $0.75 \mathrm{~W}$ & $30 \mathrm{~mJ}$ & $25 \mathrm{~Hz}$ & $300 \mu \mathrm{s}$ & $7: 7$ & EDTA-NaOCL-dw \\
Group II & IIA $(n=4)$ & $1.25 \mathrm{~W}$ & $50 \mathrm{~mJ}$ & $25 \mathrm{~Hz}$ & $300 \mu \mathrm{s}$ & $7: 7$ & $\mathrm{dw}$ \\
& IIB $(n=4)$ & $1.25 \mathrm{~W}$ & $50 \mathrm{~mJ}$ & $25 \mathrm{~Hz}$ & $300 \mu \mathrm{s}$ & $7: 7$ & EDTA-NaOCL-dw \\
Group III & IIIA $(n=4)$ & $1.75 \mathrm{~W}$ & $70 \mathrm{~mJ}$ & $25 \mathrm{~Hz}$ & $300 \mu \mathrm{s}$ & $7: 7$ & $\mathrm{dw}$ \\
& IIIB $(n=4)$ & $1.75 \mathrm{~W}$ & $70 \mathrm{~mJ}$ & $25 \mathrm{~Hz}$ & $300 \mu \mathrm{s}$ & $7: 7$ & EDTA-NaOCL-dw \\
Group IV & IVA $(n=4)$ & $2 \mathrm{~W}$ & $80 \mathrm{~mJ}$ & $25 \mathrm{~Hz}$ & $300 \mu \mathrm{s}$ & $7: 7$ & $\mathrm{dw}$ \\
& IVB $(n=4)$ & $2 \mathrm{~W}$ & $80 \mathrm{~mJ}$ & $25 \mathrm{~Hz}$ & $300 \mu \mathrm{s}$ & $7: 7$ & EDTA-NaOCL-dw \\
\hline
\end{tabular}

$\mathrm{dw}$, distilled water 
There were no areas where the smear layer was slightly removed (Fig. 2A). In the middle part of these samples, magnification images showed much lower percentages of smear layer residues. In half of the samples, a small amount of smear layer with many dentinal tubules open was observed (Fig. 2B).

While examining the efficacy of smear layer removal from middle and apical thirds, Kruskal-Wallis test revealed that none of the laser groups (IA, IIA, IIIA, IVA) was more efficient than other groups. The results for the present study showed considerable amounts of smear layer on the canal walls in the apical third, regardless of the pulse energy applied.

On the other hand, in groups IB, IIB, IIIB, IVB, samples were irrigated following an irrigation protocol (5 mL 17\% EDTA for 60 seconds, $5 \mathrm{~mL} 5 \% \mathrm{NaOCL}$, and $5 \mathrm{~mL}$ distilled water). SEM pictures revealed that there were samples where the smear layer was totally

Table 2: Frequency distribution of smear layer scores in the middle third of root canal

\begin{tabular}{lllll}
\hline & \multicolumn{4}{c}{ Smear layer removal score } \\
\cline { 2 - 5 } Groups & 0 & 1 & 2 & 3 \\
\hline Group IA & $0(0 \%)$ & 2 & $1(25 \%)$ & $1(25 \%)$ \\
Group IIA & $0(0 \%)$ & $1(25 \%)$ & $3(75 \%)$ & $0(0 \%)$ \\
Group IIIA & $0(0 \%)$ & $2(50 \%)$ & $2(50 \%)$ & $0(0 \%)$ \\
Group IVA & $1(25 \%)$ & $2(50 \%)$ & $1(25 \%)$ & $0(0 \%)$ \\
Group IB & $4(100 \%)$ & $0(0 \%)$ & $0(0 \%)$ & $0(0 \%)$ \\
Group IIB & $2(50 \%)$ & $1(25 \%)$ & $1(25 \%)$ & $0(0 \%)$ \\
Group IIIB & $2(50 \%)$ & $1(25 \%)$ & $1(25 \%)$ & $0(0 \%)$ \\
Group IVB & $1(25 \%)$ & $2(50 \%)$ & $1(25 \%)$ & $0(0 \%)$ \\
Total & 10 & 11 & 10 & 1 \\
\hline
\end{tabular}

Table 3: Frequency distribution of smear layer scores in the apical third of root canal

\begin{tabular}{lllll}
\hline & \multicolumn{4}{c}{ Smear layer removal score } \\
\cline { 2 - 5 } Groups & 0 & 1 & 2 & 3 \\
\hline Group IA & $0(0 \%)$ & $0(0 \%)$ & $1(25 \%)$ & $3(75 \%)$ \\
Group IIA & $0(0 \%)$ & $0(0 \%)$ & $2(50 \%)$ & $2(50 \%)$ \\
Group IIIA & $0(0 \%)$ & $0(0 \%)$ & $0(0 \%)$ & $4(100 \%)$ \\
Group IVA & $0(0 \%)$ & $0(0 \%)$ & $3(75 \%)$ & $1(25 \%)$ \\
Group IB & $3(75 \%)$ & $1(25 \%)$ & $0(0 \%)$ & $0(0 \%)$ \\
Group IIB & $2(50 \%)$ & $1(25 \%)$ & $1(25 \%)$ & $0(0 \%)$ \\
Group IIIB & $2(50 \%)$ & $1(25 \%)$ & $1(25 \%)$ & $0(0 \%)$ \\
Group IVB & $2(50 \%)$ & $2(50 \%)$ & $0(0 \%)$ & $0(0 \%)$ \\
Total & 9 & 5 & 8 & 10 \\
\hline
\end{tabular}
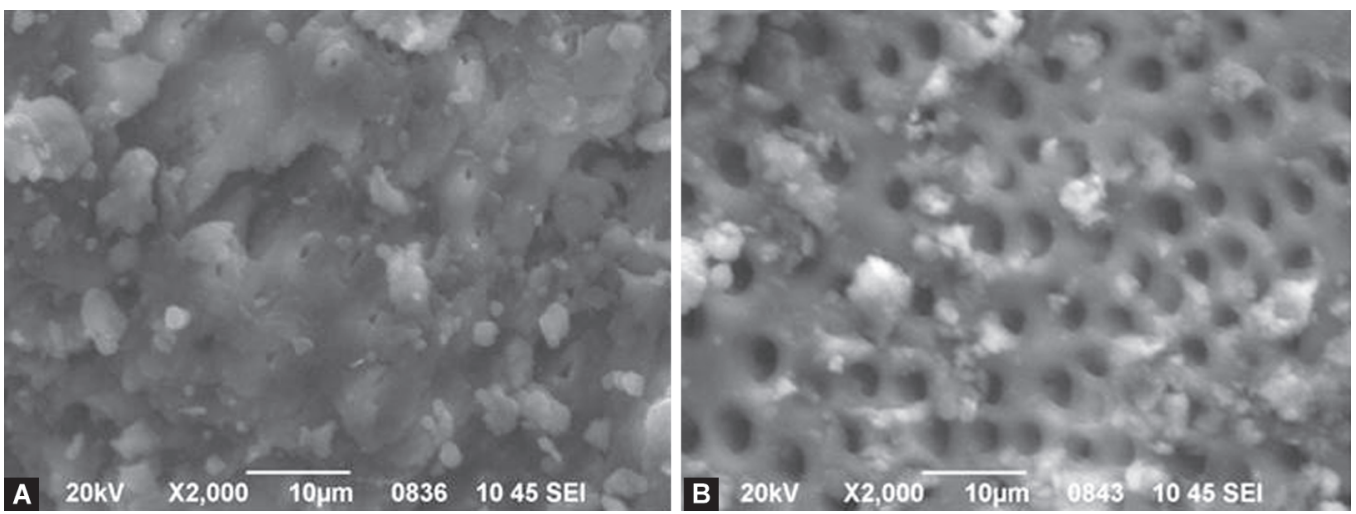

removed in apical third (Fig. 3A). In group IA (power pulse $0.75 \mathrm{~W}$ ), SEM pictures showed the best presence of smear layer removal in most samples. All the samples were evaluated with a score of 0 and dentinal tubules completely open and debris-free. In groups IIB, IIIB, IVB, there were samples free of smear layer (score 0 ), some with a small amount of smear layer (score 1), and a small percentage with homogeneous smear layer covering the root canal walls (score 2). In the middle part, in all the samples of subgroup $B$, the smear layer was completely removed or it is left a small amount of debris (Fig. 3B).

In the control group, samples were free of debris and smear layer in middle and apical part (Fig. 4).

\section{Discussion}

This study evaluated the cleanliness of dentinal walls in the middle and apical third of mesial root canals after Er:YAG laser irradiation. Laser parameters-average power and pulse energy-were compared concerning the efficiency in smear layer removal. It was supposed that there is no difference between groups irrigated with chelators and the other groups irrigated with distilled water before laser irradiation.

The smear layer is formed after the mechanical preparation of the root canal. ${ }^{18}$ There are several methods for removing the smear layer such as chemical solutions, use of ultrasonic dental devices, and dental lasers. Er:YAG laser has the optimum absorption in water which explains its increased efficiency. ${ }^{19}$ The dental tissue water content has an important role in the ablation process. ${ }^{20}$ Irradiation is absorbed by the water which is transformed into a vapor bubble. This bubble is the basis of cavitation and smear layer removal. ${ }^{21,22}$

Di Vito et al. ${ }^{23}$ studied the effectiveness of Er:YAG laser in removing smear layer using the following settings $(25 \mathrm{~mJ}, 15 \mathrm{~Hz}, 50$ $\mu$ s) with the radial and stripped tip in different irradiation times with and without the use of $17 \%$ EDTA. All irradiated groups had good results in removing the smear layer. The combination of Er:YAG laser (40 seconds irradiation time) with 17\% EDTA was the most effective. In the present study, different pulse energy settings were tested $(30-80 \mathrm{~mJ})$ in the same different irradiation times with and without the use of irrigation solutions ( $\mathrm{NaOCL}$ and EDTA).

Another study confirmed the successful removal of the smear layer using Er:YAG laser with lower power settings $(20 \mathrm{~mJ}, 10 \mathrm{~Hz}, 50$ $\mu \mathrm{s})$ for 20 or 40 seconds with $17 \%$ EDTA irrigation. ${ }^{24}$ In both previous studies, $^{23,24}$ the SEM analysis of the mentioned groups, showed open tubules, exposed intact collagen fibers, and no thermal damage to the root canal wall. The smear layer was successfully removed.

Figs $2 A$ and $B$ : Apical third $(A)$ and middle third $(B)$ after irrigation by distilled water 

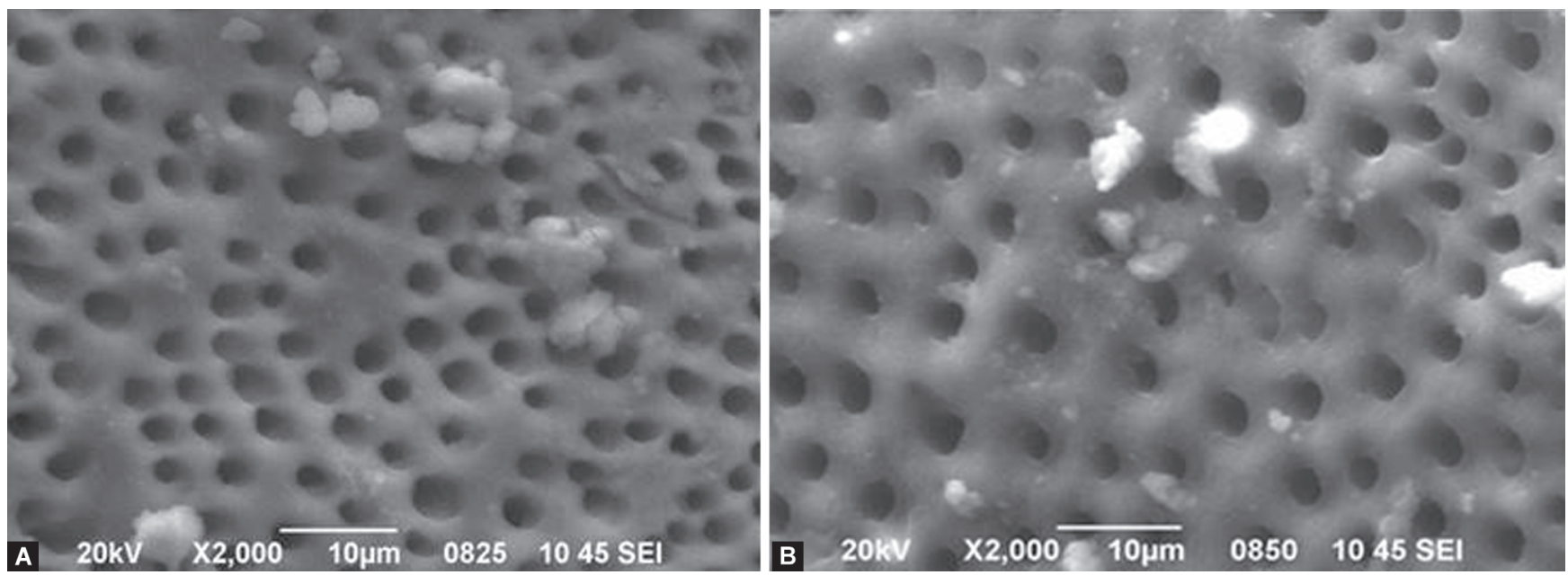

Figs $3 \mathrm{~A}$ and $\mathrm{B}$ : Apical third (A) and middle third (B) after irrigation by $17 \%$ EDTA and $5 \% \mathrm{NaOCL}$

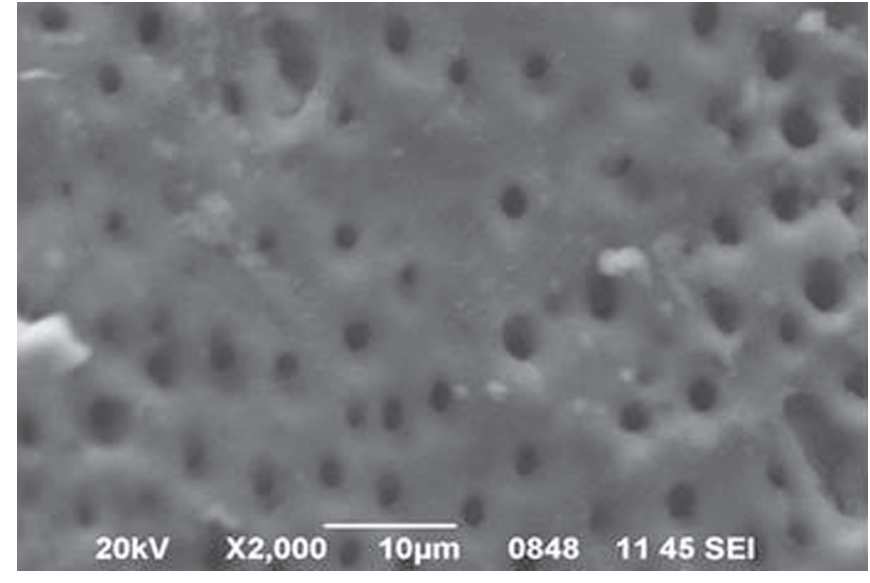

Fig. 4: Apical third in control group

The sample of this study consisted of mesial roots of first mandibular molars. It is now well accepted that the mandibular first molar exhibits several anatomical variations not only in the number of roots but also in canal morphology. ${ }^{25,26}$ The new lanced endodontic tip R300T tip is used for the first time for smear layer removal from molars. Herrmann ${ }^{27}$ supported that after root canal preparation with mechanical nickel-titanium instrumentation, $\mathrm{R} 300 \mathrm{~T}$ ( $50 \mathrm{~mJ}, 25 \mathrm{pps}$ ) can be used. A recent in vitro study ${ }^{28}$ aimed at assessing the antimicrobial activity of Er:YAG laser $(70 \mathrm{~mJ}, 20$ pps) and R300T on several microorganisms relative to persistent endodontic infection.

Laser applications necessitate root preparation at least size 30. However, R300T of $300 \mu \mathrm{m}$ necessitates a larger root canal diameter than ISO 30. In our studies, in subgroups A, it was very difficult the instrumentation of such narrow and curved root canals without a chelator. Many samples were rejected due to a broken file.

Ramalho et al. ${ }^{29}$ showed that the Er:YAG laser did not remove the smear layer from dentinal wall surfaces, because they were not reachable by its optical fiber. Better results have been reported when comparing Er:YAG with other methods of smear layer removal. Er:YAG is reported to have the most effective removal of smear layer from the dentine wall compared to $17 \%$ EDTA, $6 \%$ citric acid, $6 \%$ phosphoric acid, and $\mathrm{CO}_{2}$ laser. ${ }^{9}$ In another study, Er:YAG had also shown to have better smear layer removal when compared to other wavelengths such as Argon and Nd:YAG lasers. ${ }^{7}$

Irrigation with EDTA was used in this study. Chelating agents have been suggested for removing the smear layer, including EDTA, citric acid, and orthophosphoric acid. ${ }^{30}$ These irrigants dramatically improve the cleaning ability of root canals. On the other hand, the combination of $\mathrm{NaOCL}$ and EDTA removed the smear layer only partially ${ }^{31}$ and unable to clean in the apical portion of the root canals, and hence, to improve that in apical portion laseractivated systems were used in the recent study of Dhawan et al. ${ }^{32}$ Comparing the two major groups from a clinical point of view, it can be concluded that Er:YAG laser is more effective in combination with EDTA solution. Er:YAG laser adequately cleaned dentinal walls at the middle third. The absence of the chelating factor played an important role in the laser mechanism of smear layer removal in the apical third.

Pulse duration has been found to influence ablation. ${ }^{33-35}$ Shorter pulse durations lead to less thermal damage to surrounding tissue and results in more efficient ablation. ${ }^{36}$ Unfortunately, the device used has a constant pulse duration of $300 \mu \mathrm{s}$ which leads to smaller peak power values.

Er:YAG laser due to its high water absorption and hydroxyapatite has low penetration into the root walls. ${ }^{37}$ Its antimicrobial action is limited in removing smear layer and superficially cleaning the dentinal walls better than conventional methods, but has significantly less transmission in dentine than a near-infrared diode laser. ${ }^{38}$

\section{Conclusion}

The outcome of the present study showed that laser-assisted smear layer removal with Er:YAG laser at four different energy pulses in combination with EDTA showed to a great degree, effectiveness in smear layer removal. The presence of the chelating factor played an important role in the mechanism of smear layer removal. Further research needs to be performed to find the optimal irradiation protocol (average power, pulse energy, pulse duration) for the laser systems, which could remove the smear layer completely. 


\section{References}

1. Fried D, Zuerlein M, Featherstone JDB, et al. IR laser ablation of dental enamel: mechanistic dependence on the primary absorber. App Surf Sci 1998;129:852-856. DOI: 10.1016/S0169-4332(97)00755-1.

2. Zuerlein MJ, Fried D, Featherstone JDB, et al. Optical properties of dental enamel in the mid-IR determined by pulsed photothermal radiometry. leee J Select Topics Quantum Electron 1999;5(4):10831089. DOI: $10.1109 / 2944.796333$.

3. Hibst R. Lasers for caries removal and cavity preparation: state of the art and future directions. J Oral Laser Appl 2002;2(4):203-212.

4. Hibst R, Keller U. Experimental studies of the application of the Er:YAG laser on dental hard substances. I. Measurement of the ablation rate. Lasers Surg Med 1989;9(4):338-344. DOI: 10.1002/lsm.1900090405.

5. Lukac M, Marincek M, Grad L, et al. Er:YAG pulses for fast and precise cavity preparation. J Oral Laser Appl 2004;4:171-173.

6. Baraba A, Miletic I, Jukic Krmek A, et al. Ablative potential of the erbium-doped yttrium aluminium garnet laser and conventional handpieces: a comparative study. Photomed Laser Surg 2009;27(6):921-927. DOI: 10.1089/pho.2008.2416.

7. Takeda FH, Harashima T, Kimura Y, et al. Comparative study about the removal of smear layer by three types of laser devices. J Clin Laser Med Surg 1998;16(2):117-122. DOI: 10.1089/clm.1998.16.117.

8. Takeda FH, Harashima T, Kimura Y, et al. Efficacy of Er: YAG laser irradiation in removing debris and smear layer on root canal walls. J Endod 1998;24(8):548-551. DOI: 10.1016/S0099-2399(98)80075-7.

9. Takeda FH, Harashima T, Kimura Y, et al. A comparative study of the removal of smear layer by three endodontic irrigants and two types of laser. Int Endod J 1999;32(1):32-39. DOI: 10.1046/j.13652591.1999.00182.x.

10. Pecora JD, Brugnera-Junior A, Cussioli AL, et al. Evaluation of dentin root canal permeability after instrumentation and Er: YAG laser application. Laser Surg Med 2000;26(3):277-281. DOI: 10.1002/ (sici)1096-9101(2000)26:33.0.co;2-m.

11. Fried D. IR laser ablation of dental enamel. In: Featherstone JDB, Rechmann P, Fried D, ed. Lasers in Dentistry VI. Volume Proc. SPIE, vol. 3910 2000. pp. 136-148.

12. Seka W, Featherstone JDB, Fried D, et al. Laser ablation of dental hard tissues from explosive ablation to plasma mediate ablation. In: Wigdor HA, Featherstone JD, White JM, et al., ed. Lasers in Dentistry II. Proc SPIE, vol. 2672 1996. pp. 144-158.

13. Vertucci F, Seelig A, Gillis R. Root canal morphology of the human maxillary second premolar. Oral Surg Oral Med Oral Pathol 1974;58(3):456-464. DOI: 10.1016/0030-4220(74)90374-0.

14. Al-Nazhan S. Incidence of four canals in root-canal-treated mandibular first molars in a Saudi Arabian sub-population. Int Endod J 1999;32(1):49-52. DOI: 10.1046/j.1365-2591.1999.00188.x.

15. Al-Qudah AA, Awawdeh LA. Root and canal morphology of mandibular first and second molar teeth in a Jordanian population. Int Endod J 2009;42(9):775-784. DOI: 10.1111/j.1365-2591.2009.01578.x.

16. Wang $Y$, Zheng Q-H, Zhou X-D, et al. Evaluation of the root and canal morphology of mandibular first permanent molars in a western Chinese population by cone beam computed tomography. J Endod 2010;36(11):1786-1789. DOI: 10.1016/j.joen.2010.08.016.

17. de Pablo OV, Estevez R, Peix Sanchez M, et al. Root anatomy and canal configuration of the permanent mandibular first molar: a systematic review. J Endod 2010;36(12):1919-1931. DOI: 10.1016/j. joen.2010.08.055.

18. Mader CL, Baumgartner JC, Peters DD. Scanning electron microscopic investigation of the smeared layer on root canal walls. J Endod 1984;10(10):477-483. DOI: 10.1016/S0099-2399(84)80204-6.

19. Diaci J, Gaspirc B. Review comparison of Er: YAG and Er, Cr: YSGG lasers used in dentistry. J Laser Health Acad 2012. 1.
20. Armengol V, Jean A, Rohanizadeh R, et al. Scanning electron microscopic analysis of diseased and healthy dental hard tissues after Er:YAG laser irradiation: in vitro study. J Endod 1999;25(8):543-546. DOI: 10.1016/S0099-2399(99)80376-8.

21. Brugnera AJR, Zanin F, Barbin EL, et al. Effects of Er:YAG and Nd:YAG laser irradiation on radicular dentine permeability using different irrigating solutions. Lasers Surg Med 2003;33(4):256-259. DOI: 10.1002/lsm.10214.

22. Kivanc BH, Arisu HD, Ozcan S, et al. The effect of the application of gaseous ozone and Nd: YAG laser on glass-fibre post bond strength. Aust Endod J 2012;38(3):118-123. DOI: 10.1111/j.17474477.2010.00265.x.

23. Di Vito E, Colonna MP, Olivi G. The photoacoustic efficacy of an Er:YAG laser with radial and stripped tips on root canal dentin walls: an SEM evaluation. J Laser Dentis 2011;19(1):156-161.

24. DiVito E, Lloyd A. Er: YAG laser for 3-dimensional debridement of canal systems: use of photon-induced photoacoustic streaming. Dent Today 2012;31(122):124-127.

25. Prabhu NT, Munshi AK. Additional distal root in permanent mandibular first molars: report of a case. Quintess Int 1995;26(8):567569.

26. Schumann C. Endodontic treatment of a mandibular first molar with radix entomolaris: a case report. Endo (Lond Engl) 2008;2:301-304.

27. Herrmann $\mathrm{H}-\mathrm{W}$. Using the AdvErL Evo laser for endodontic treatments. Laser 2017;3:20-23.

28. Henninger E, Berto LA, Eick S, et al. In vitro wffect of Er:YAG laser on different single and mixed microorganisms being associated with endodontic infections. Photobiomodulat, Photomed, Laser Surg 2019;37(6):369-375. DOI: 10.1089/photob.2018.4557.

29. Ramalho KM, Marques MM, Apel C, et al. Morphological analysis of root canal walls after Er:YAG and Nd:YAG laser irradiation: a preliminary SEM investigation. JOLA 2005;5(2):91-96.

30. Perez $F$, Calas $P$, Rochd $T$. Effect of dentin treatment on in vitro root tubule bacterial invasion. Oral Surg Oral Med Oral Pathol 1996;82(4):446-451. DOI: 10.1016/s1079-2104(96)80313-1.

31. Ciucchi B, Khettabi M, Holz J. The effectiveness of different endodontic irrigation procedures on the removal of the smear layer: a scanning electron microscopic study. Int Endod J 1989;22(1):21-28. DOI: 10.1111/j.1365-2591.1989.tb00501.x.

32. Dhawan $\mathrm{S}$, Jasuja $\mathrm{P}$, Khurana $\mathrm{H}$, et al. A comparative evaluation of the efficacy of erbium: yttrium-aluminum-garnet and diode lasers in smear layer removal and dentin permeability of root canal after biomechanical preparation - a scanning electron microscopy study. J Indian Soc Pedod Prev Dent 2020;38(1):64-70. DOI: 10.4103/JISPPD. JISPPD_174_19.

33. Meister J, Apel C, Franzen R, et al. Influence of the spatial beam profile on hard tissue part I: multimode emitting Er:YAG lasers. Lasers Med Sci 2003;18(2):112-118. DOI: 10.1007/s10103-003-0263-9.

34. Meister J, Franzen R, Apel C, et al. Multireflection pumping concept for miniaturized ablation diode-pumped solid-state lasers. Appl Opt 2004;43(31):5864-5869. DOI: 10.1364/ao.43.005864.

35. Apel C, Franzen R, Meister J, et al. Influence of the pulse duration of an Er:YAG laser system on the ablation threshold of dental enamel. Lasers Med Sci 2002;17(4):253-257. DOI: 10.1007/s101030200037.

36. Meister J, Franzen R, Forner $\mathrm{K}$, et al. Influence of the water content in dental enamel and dentin on ablation with erbium YAG and erbium YSGG lasers. J Biomed Opt 2006;11(3):34030. DOI: 10.1117/1. 2204028.

37. Torabinejad $M$, Khademi AA, Babagoli J, et al. A new solution for the removal of the smear layer. J Endod 2003;29(3):170-175. DOI: 10.1097/00004770-200303000-00002.

38. Gutnecht N. Lasers in endodontics. J Laser Health Acad 2008. 4. 